CONVECTIVE OVERSHOOTING: THE UPPER MASS LIMIT FOR STARS UNDERGOING

CORE HE-FLASH

G. Bertelli ${ }^{1,2}$ and A. Bressan 3
1) National Council of Research of Italy, C.N.R. - G.N.A.
2) Institute of Astronomy, Padova, Italy
3) International School for Advanced Studies, Trieste, Italy

Theoretical Rationale

With classic models of stars, in which overshooting from convective cores is not taken into account, the transition mass, Mtr, separating stars which undergo core He-flash from stars which ignite He-burning nonviolently in a non degenerate core, is set around 2.2 M॰ (Iben 1967) for Pop I chemical composition $(X=0.700, Z=0.020)$. This value is known however to depend on the chemical abundances, being lower at increasing $Y$ and decreasing Z (Wagner 1974; Sweigart and Gross 1978). However, in recent years many independent arguments have indicated that convective overshooting from the central cores may play an important role in stellar evoluion (Bertelli et al 1985). In order to assess the dependence of Mtr on overshooting, we have computed evolutionary sequences of $1.4 \mathrm{M}, 1.5$ M॰ and 1.6 M॰ with chemical composition $X=0.700$ and $Z=0.020$ adopting the description of convective overshooting formulated by Bressan et al (1981) for their parameter $\lambda=1 / \mathrm{Hp}=1$. As shown in the $\mathrm{T}_{c}$ vs $\rho_{c}$ diagram of Fig 1, while the tracks of $1.4 \mathrm{M} \odot$ and $1.5 \mathrm{M} \odot$ stars deeply penetrate into the region of high degeneracy and likely undergo core He-flah, the $1.6 \mathrm{M} \odot$ star succeeds in igniting helium in non degenerate conditions. This means that the classic value of 2.2 Mo for $\mathrm{Mtr}$ is now lowered to the mass range 1.6 t॰ $1.5 \mathrm{M}$. The HR diagram of the $1.4 \mathrm{M}, 1.5 \mathrm{M} \odot$ and $1.6 \mathrm{M} \odot$ stars in presence of convective overshooting is shown in Fig 2. The region of stationary core He-burning for the $1.6 \mathrm{M} \odot$ and the extension of the red giant branches for the $1.4 \mathrm{M} \odot$ and 1.5 Mo stars are also indicated.

\title{
Observational Counterpart
}

The luminosity function of evolved stars of clusters with age in the range 1 to $2 \times 10^{9}$ yr may constitute a powerful test on the actual value for Mtr. In fact, the luminosity function of red stars is expected to suddenly change passing from clusters having a turn-off mass lower than Mtr to clusters with a turn-off mass greater than this. In the recent study by Barbaro and Pigatto (1984) of a large number of old galactic open clusters with about solar metallicity, it has been suggested that the above transition should occur at approximately $1.5 \mathrm{M}$ (instead of the classic 2.2 Mo. 421

C. W. H. De Loore et al. (eds.), Luminous Stars and Associations in Galaxies, 421-422.

(C) 1986 by the IAU. 
Those authors indicated in convective overshooting during the core H-burning phase the way out of the above disagreement between standard theory and observation. This is strongly supported by the present evolutionary computations. In addition to this, since the dependence of Mtr on overshooting during the core H-burning phase, is not biased by other uncertainties affecting subsequent evolution (neutrino cooling and semiconvection and/or overshooting during the core He-burning phase), this finding may be used to infer the mass size of convective cores, hence the efficiency of convective overshooting, in real stars.

A more complete description of the above results will be published elsewhere (Astron. Astrophys.).

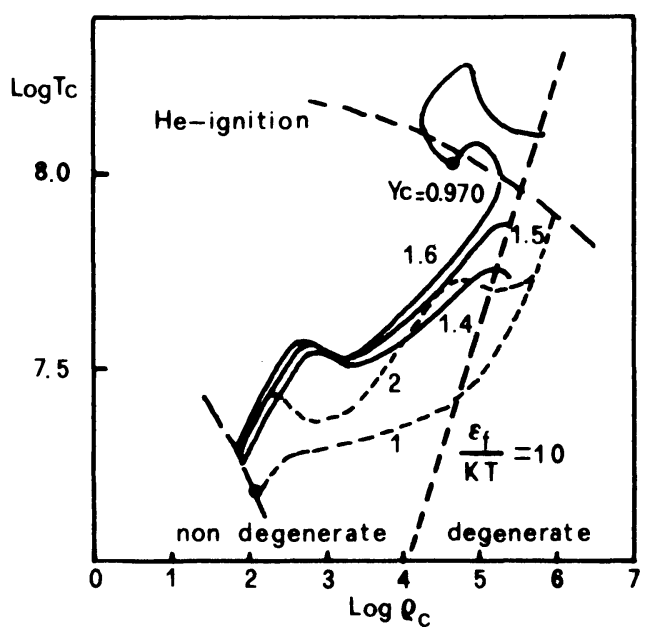

Fig $1 \quad T_{c}$ vs $\rho_{c}$ diagram for models with (solid line) and without (broken line) overshooting

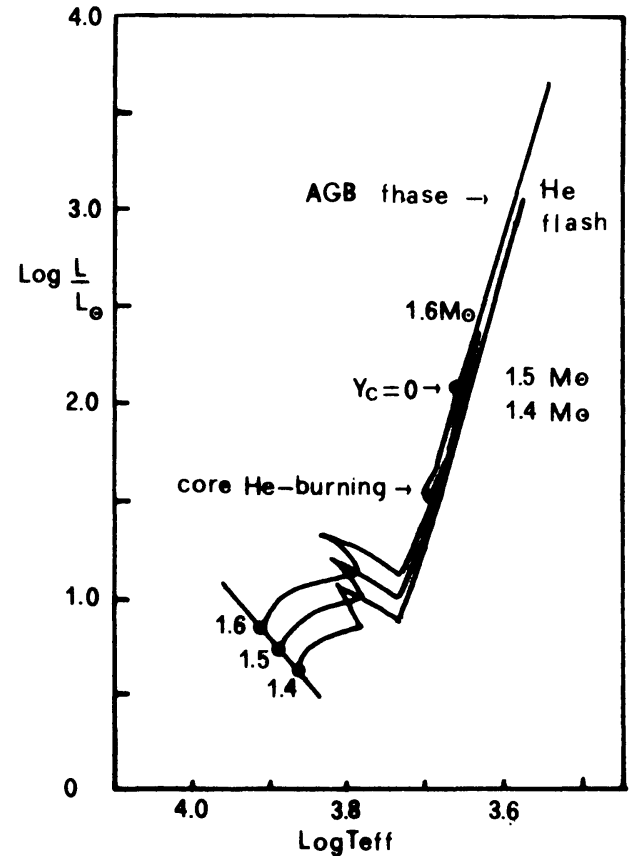

Fig 2 The HR diagram for models with overshooting

\section{References}

Barbaro, G., Pigatto, L., 1984, Astron. Astrophys., 136, 355

Bertelli, G., Bressan, A., Chiosi, C., 1985, Astron. Astrophys., in press Bressan, A., Bertelli, G., Chiosi, C., 1981, Astron. Astrophys., 102, 25 Iben, I. Jr., 1967, Ann. Rev. Astron. Astrophys., 5, 571 Sweigert, A. V., Gross, P. G., 1978, Astrophys. J., 36, 405 Wagner, R. L., 1974, Astrophys. J., 191, 173 